\title{
Neural Correlates of Successful and Unsuccessful Verbal Memory Encoding
}

\author{
Daniel J. Casasanto, $*$ William D. S. Killgore, ${ }^{*} \dagger$ Joseph A. Maldjian, $\ddagger$ Guila \\ Glosser,* David C. Alsop, $\neq$ Ayanna M. Cooke,* Murray Grossman,* \\ and John A. Detre*, \\ Departments of *Neurology and $¥$ Radiology, University of Pennsylvania Medical Center \\ and Brain Imaging Center, †Harvard Medical School
}

Published online February 14, 2002

\begin{abstract}
Recent neuroimaging studies suggest that episodic memory encoding involves a network of neocortical structures which may act interdependently with medial temporal lobe (mTL) structures to promote the formation of durable memories, and that activation in certain structures is modulated according to task performance. Functional magnetic resonance imaging (fMRI) was used to determine the neural structures recruited during a verbal episodic encoding task and to examine the relationship between activation during encoding and subsequent recognition memory performance across subjects. Our results show performance-correlated activation during encoding both in neocortical and medial temporal structures. Neocortical activations associated with later successful and unsuccessful recognition memory were found to differ not only in magnitude, but also in hemispheric laterality. These performance-related hemispheric effects, which have not been previously reported, may correspond to betweensubject differences in encoding strategy. ๑ 2002 Elsevier Science (USA)
\end{abstract}

Key Words: functional imaging; episodic memory; encoding; hemispheric effects.

\section{INTRODUCTION}

Episodic memory encoding is the process by which the experience of an event is transformed into a memory trace that is available for conscious recollection (Tulving et al., 1994). Positron emission tomography (PET) and functional magnetic resonance imaging (fMRI) studies have reported activation associated with verbal episodic encoding in a widely distributed network of brain regions that includes the prefrontal, temporal, parietal, occipital, and cerebellar cortices as well as the hippocampal formation (Tulving, Habib, Nyberg, Lepage, \& McIntosh, 1999; Wagner et al., 1998), yet it is unclear to what extent activation in these regions predicts whether experienced events will be remembered. The majority of functional imaging studies of memory have sought to identify the neural structures that subserve particular memory processes. Recently, however, functional imaging has also been used to investigate how activation in these structures varies according to the success of a subject's task performance.

We gratefully acknowledge the contribution of Dr. Jonathan Raz and dedicate this report to his memory. This project was supported by NIH Grants NS37488 and NS02079.

Address correspondence and reprint requests to John A. Detre, Department of Neurology, University of Pennsylvania Medical Center, 3400 Spruce Street, Gates Building 3 West, Philadelphia, PA 19104. Fax: (215) 614-1927. E-mail: detre@mail.med.upenn.edu. 
There is considerable disagreement among the initial fMRI studies that have examined the relationship between activation during verbal episodic encoding and subsequent memory performance. (For a complete review including PET and ERP literature on subsequent memory effects, see Wagner et al., 1999.) Using a blocked-trial word list learning paradigm, Fernandez et al. (1998) found positive within-subject correlations between the number of successfully recalled words and the intensity of activation during encoding in the bilateral posterior hippocampus, but not in surrounding mesial temporal lobe (mTL) structures that have also been implicated in declarative memory encoding (Fernandez et al., 1998). In a subsequent experiment, Fernandez et al. (1999) found a correlation between word recall and sustained activation during encoding in the bilateral entorhinal cortex, but not the hippocampus (Fernandez et al., 1999). Wagner et al. (1998) used an event-related paradigm that allowed direct comparison of encoding trials resulting in successful and unsuccessful word recognition, which showed that the left prefrontal cortex, parahippocampus, and fusiform gyrus were more active during encoding of words later remembered than words later forgotten (Wagner et al., 1998). The finding of performance-correlated prefrontal activation differs from that of the 1999 Fernandez et al. study, which showed prefrontal activation during word encoding that did not correlate significantly with subsequent word recall. Also, in the study by Wagner et al. hippocampal activation was not found to correlate with success of encoding, in contrast with Fernandez et al. (1998). A more recent study by Kirchhoff et al. (2000) found no verbal subsequent memory effects that were significant at the voxel level in either the prefrontal cortex or MTL, although both regions were activated during word encoding (Kirchhoff, Wagner, Maril, \& Stern, 2000). Some of these discrepancies may be attributable to variations in experimental design. For example, the studies by Fernandez et al. used blocked trials and required intentional encoding of word lists, whereas the studies by Wagner et al. and Kirchhoff et al. used event-related designs and induced incidental encoding of single words. Nevertheless, it is clear that further experimentation is needed to elucidate the relationship between functional activation during verbal encoding and subsequent memory performance.

In the present study, fMRI was used to examine neural activity during intentional encoding of verbal stimuli. Subjects viewed blocks of four-word declarative sentences alternating with matched blocks of nonverbal control strings and were instructed to remember the sentences for a postscan recognition memory test. The goal of the experiment was to determine the relationship between encoding success and functional activation across subjects and to compare the locations of brain regions in which activation was performance-invariant with those in which activation was modulated by task performance. Two successive analyses were performed. Cognitive subtraction was used to identify brain structures recruited during sentence encoding, and correlational analysis was used to reveal how activation during encoding varied according to subjects' performance on the postscan recognition test, which served as an index of encoding success.

\section{MATERIALS AND METHODS}

\section{Subjects}

Eleven healthy, normal volunteers (five men and six women) between the ages of 18 and 30 years were recruited from the University of Pennsylvania community and paid \$20 for their participation. All subjects were students or trainees at the University, were native speakers of English, and were righthanded by self-report. Informed consent was obtained from each subject prior to participation in the study, which was approved by the University of Pennsylvania Institutional Review Board. 


\section{Task Design}

Subjects viewed a total of 60 novel stimuli, presented over six 40 -s blocks (10 stimuli per block, 3500-ms presentation, 500-ms ISI) while lying supine in the bore of the MRI scanner. Stimulus blocks alternated with control blocks during which a single control image was presented repeatedly. Stimuli were four-word, active, declarative sentences composed of words culled from children's books estimated to be at the fifth-grade reading level and presented in Chicago 24-point font (e.g., They buried the money and The cat has fleas). Simple sentences were chosen so that this task could be administered to neurologically impaired patients with cognitive deficits, although all data reported presently pertain to healthy subjects. The control image, matched with the target stimuli for size, luminosity, and presentation rate, was a set of four strings composed of asterisks, of the same mean length as the stimulus words. Stimulus presentation routines were developed on a Macintosh Powerbook (Apple Computer, Cupertino, CA) using Psyscope software (Cohen, MacWhinney, Flatt, \& Provost, 1993). Stimuli were backprojected using an Epson LCD projector (Model No. ELP-5000) onto a viewing screen positioned approximately 7 feet from the subject's eyes, which was easily visible via a mirror mounted in the scanner head coil. Subjects were instructed to remember the stimuli for a recognition test immediately following the encoding task and to attend to the control images, but not to memorize them.

For the recognition test, subjects viewed all 60 of the stimuli presented during encoding, randomly intermixed with an equal number of novel distractors. While still lying in the scanner bore, subjects were required to distinguish studied target sentences from unstudied distractors and to respond using a button box interfaced with the Macintosh computer via fiberoptic cable. The old-new recognition test was self-paced, and subjects were informed that both the speed and accuracy of their responses would be recorded. Functional imaging data were collected during encoding, but not during recognition testing.

\section{Image Acquisition}

Imaging data were collected on a 1.5-tesla GE Horizon Echospeed MRI using the product quadrature radiofrequency head coil. Foam padding was used to comfortably restrict head motion. Sagittal and axial T1-weighted structural images were obtained for each subject. Prior to functional activation, data were acquired for correction of image distortion due to static susceptibility effects (Alsop, 1995). Gradient echo, echoplanar images sensitive to BOLD contrast $(\mathrm{TR}=2000 \mathrm{~ms}$, TEeff $=50 \mathrm{~ms})$ were then obtained in 18 to 20 contiguous 5 -mm-thick axial slices, using a $24 \times 15 \mathrm{~cm}$ field of view with a $64 \times 40$ acquisition matrix, resulting in a nominal pixel resolution of $3.75 \times 3.75 \times 5 \mathrm{~mm}$. Functional activation was measured over a single 240-scan run consisting of 680 -s task/control cycles.

\section{Image Processing and Data Analysis}

Recognition test performance was assessed by computing a discriminability index for each subject $[$ Discriminability $=(\%$ hits $)-(\%$ false alarms $)]$. Discriminability scores were entered into subsequent analyses as a measure of encoding success.

Raw image data were reconstructed offline on SUN UltraSparc workstations (SUN Microsystems, Mountain View, CA) using software developed in Interactive Data Language (IDL, Research Systems, Boulder, CO). Raw data were corrected for static susceptibility-induced distortions on the basis of data acquired during phase-encoded reference scanning (Alsop, 1995). Statistical parametric maps (SPMs) were generated for each subject's encoding run using SPM99 software (Wellcome Department of Cognitive Neurology, London, UK) implemented in Matlab (The Mathworks Inc. Natick, MA) with an IDL interface. The T1-weighted images were normalized to a standard template in Montreal Neurological Institute (MNI) coordinate space within SPM99. The functional data sets were motion corrected (intrarun realignment) within SPM99 using the first image as the reference. The functional data sets were normalized to MNI space using image header information to determine the 16-parameter affine transform between the functional data sets and the T1-weighted images (Maldjian et al., 1997), in combination with the transform computed within SPM99 for the T1-weighted anatomic images to MNI space. The normalized data sets were resampled to $4 \times 4 \times 4 \mathrm{~mm}$ within MNI space using sync interpolation. A second realignment step (interrun realignment) was then performed between successive normalized runs within each subject, using the initial normalized run as the target. This was done to eliminate motion between the successive runs within each subject. The data sets were smoothed using a $8 \times 8 \times 10 \mathrm{~mm}$ Full Width at Half Maximum Gaussian smoothing kernel, and SPMs were generated using the general linear model within SPM99. A 6-s time-shifted box-car waveform was used as the reference paradigm, and the ANCOVA model with global activity as a confound was employed for the statistical analysis. Temporal smoothing, detrending, and high-pass filtering were performed as part of the SPM analysis. Intrinsic 
correlations were removed by estimating the autocorrelation structure using an AR(1) first order autoregressive equation.

Two separate second-level analyses were performed: a functional localization analysis and a brainbehavior correlation analysis. Localization of group activation associated with stimulus encoding was determined using the SPM99 random effects model (Holmes \& Friston, 1998), with the individual subjects' contrast maps as input. The same input data were analyzed using individual subjects' discriminability scores as a behavioral covariate. This yielded a random effects model group SPM with two contrasts, the first showing regions of activation that correlated positively, and the second showing regions of activation that correlated negatively with subjects' discriminability scores.

Results of both the functional localization and brain-behavior analyses were examined in two ways. Whole-brain multisubject SPMs were viewed using a height threshold of $\alpha=.01$ and cluster corrected at $p<.05$, resulting in a minimum cluster size of 51 contiguous voxels, thus reducing the likelihood of false positive activations (type I error). Because this excluded potentially significant clusters of activation with lesser spatial extent, a focused region of interest (ROI) analysis was also conducted to detect activation in the mesial temporal lobe (mTL), where smaller clusters were expected based on our prior experience (Casasanto, Killgore, Glosser, Maldjian, \& Detre, 2000). The height threshold was set to $\alpha=.05$, with no additional correction. The mTL ROI comprised the hippocampus, parahippocampus, and fusiform gyrus. Anatomical regions were defined using the SPL/NSL Anatomy Browser (Kikinis et al., 1996), which was previously normalized to the MNI SPM template for use with our fMRI data. Active suprathreshold voxels in each lateralized region were quantified using software developed in IDL and interfaced with SPM99 software.

\section{RESULTS}

\section{Behavioral Results}

Performance on the postscan recognition test confirmed that subjects encoded target stimuli satisfactorily. Subjects' mean discriminability score was $0.70(S D \pm .17)$, significantly above chance $[t(11)=13.97, p=.0001]$.

\section{Imaging Results}

Whole-brain data from the cognitive subtraction analysis showed suprathreshold activation $(\alpha=.01$, cluster level significance, $p<.05)$ associated with sentence encoding in the left-hemisphere inferior prefrontal and middle temporal gyri and in the right-hemisphere postcentral and superior temporal gyri (see Fig. 1, top row). Whole-brain data from the brain-behavior correlation analysis showed suprathreshold activation correlating positively with recognition memory performance in the left-hemisphere inferior prefrontal, supramarginal, and middle temporal gyri (see Fig. 1 , middle row) and correlating negatively with recognition memory performance in the right-hemisphere superior temporal gyrus and sylvian fissure (see Fig. 1, bottom row).

Data in the mTL ROI for the cognitive subtraction analysis showed suprathreshold activation $(\alpha=.05)$ bilaterally in the hippocampus (left $>$ right, $n s)$, parahippocampus (right $>$ left, $n s$ ), and the right fusiform gyrus. MTL ROI data for the brainbehavior correlation analysis showed suprathreshold activation correlating positively with recognition memory performance bilaterally in the hippocampus, parahippocampus, and fusiform gyrus. The proportion of active suprathreshold voxels was greater in the left hemisphere than the right hemisphere for all three anatomical structures; however, the differences were not statistically significant based on a $z$ test for the independence of two proportions (Hinkle, Wiesrsma, \& Jurs, 1988). No suprathreshold activation was found in the MTL ROI correlating negatively with recognition memory performance. (See Table 1.) 

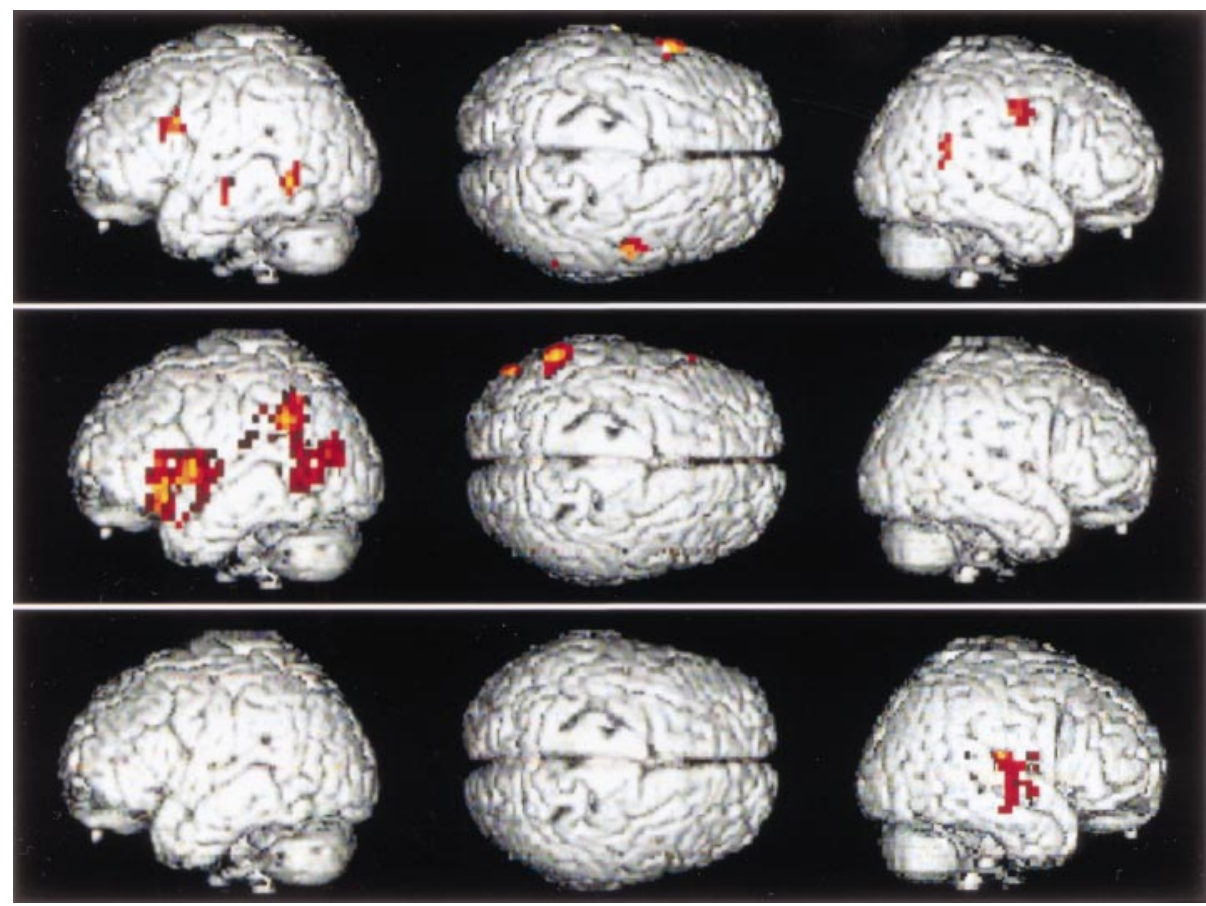

FIG. 1. Group statistical parametric maps $(n=11)$ show whole-brain activation. Regions exceeding a statistical threshold $(\alpha=.01$, cluster level significance $p<.05)$ are displayed in a red-to-yellow color scale indicating increasing significance. (Top row) Cognitive subtraction (task-control) reveals bilateral activation associated with sentence encoding in the left-hemisphere inferior prefrontal cortex (BA 44), anterior middle temporal gyrus (BA 22), and posterior middle temporal gyrus (BA 37) and in the righthemisphere postcentral gyrus (BA 40) and superior temporal gyrus (BA 39). (Middle row) Unilateral activation correlating positively with recognition memory performance in the left-hemisphere inferior prefrontal cortex (BA 44/45), supramarginal gyrus (BA 40), and posterior middle temporal gyrus (BA 37). (Bottom row) Unilateral activation correlating negatively with recognition memory performance in the right-hemisphere superior temporal gyrus (BA 41/42) and sylvian fissure.

\section{DISCUSSION}

Distinct patterns of activation during sentence encoding were associated with relatively good and poor subsequent memory performance. Better performance during recognition testing was associated with activation in left-hemisphere prefrontal and temporoparietal structures and in bilateral mTL structures; worse performance was associated with activation in right-hemisphere temporoparietal structures, but not with any mTL activation. These findings corroborate results of previous studies indicating that areas of the left prefrontal cortex and bilateral mTL mediate encoding processes for verbal stimuli that facilitate their subsequent retrieval (Fernandez et al., 1998, 1999; Wagner et al., 1999). Temporoparietal activation has been reported in previous verbal encoding studies (Kirchhoff et al., 2000; Wagner et al., 1999), but it has not been found previously to correlate with subsequent recognition memory performance. The present findings suggest that not only the magnitude but also the hemispheric laterality of activation in temporoparietal structures may be performance-correlated. The hemispheric effects of performance observed in temporoparietal structures upon correlational analysis were not found in the mTL. Furthermore, no strong hemispheric effects were found upon cognitive subtraction analysis; rather, bilateral activation was associated with sentence encoding in both the neocortex and the mTL. Compared with activations found in the cognitive sub- 
TABLE 1

Volume and Significance of Activation in MTL ROI

\begin{tabular}{lrrrcc}
\hline \multicolumn{1}{c}{ Anatomical region } & $\begin{array}{c}\text { Total } \\
\text { volume }\end{array}$ & $\begin{array}{c}\text { Active } \\
\text { volume }\end{array}$ & $\begin{array}{c}\text { Mean } \\
Z\end{array}$ & $\begin{array}{c}\text { Maximum } \\
Z\end{array}$ & $\begin{array}{c}\text { Stereotaxic } \\
\text { (MNI) coordinates }\end{array}$ \\
\hline Cognitive subtraction (sentences - asterisks) & 71 & 7 & 2.61 & 3.31 & $(-28-445)$ \\
$\quad$ Left hippocampus & 78 & 4 & 2.38 & 2.55 & $(-24-12-25)$ \\
Left parahippocampus & 148 & 0 & - & - & - \\
Left fusiform gyrus & 75 & 1 & 2.18 & 2.18 & $(16-20-25)$ \\
Right hippocampus & 81 & 12 & 2.45 & 3.31 & $(24-40-20)$ \\
Right parahippocampus & 133 & 4 & 2.51 & 3.03 & $(20-48-15)$ \\
Right fusiform gyrus & & & & & \\
Positive brain-behavior correlation & 71 & 11 & 2.76 & 6.55 & $(-32-400)$ \\
$\quad$ Left hippocampus & 78 & 20 & 2.38 & 6.33 & $(-24-44-10)$ \\
Left parahippocampus & 148 & 17 & 2.60 & 5.80 & $(-40-28-20)$ \\
Left fusiform gyrus & 75 & 5 & 2.75 & 3.13 & $(32-20-25)$ \\
Right hippocampus & 81 & 10 & 2.99 & 6.33 & $(24-36-20)$ \\
Right parahippocampus & 133 & 12 & 2.00 & 2.78 & $(36-20-30)$ \\
$\quad$ Right fusiform gyrus & 71 & 0 & - & - & - \\
Negative brain-behavior correlation & & & & - & - \\
$\quad$ Left hippocampus & 78 & 0 & - & - & - \\
Left parahippocampus & 148 & 0 & - & - & - \\
Left fusiform gyrus & 75 & 0 & - & - & - \\
Right hippocampus & 81 & 0 & - & - & - \\
Right parahippocampus & 133 & 0 & - & - & - \\
Right fusiform gyrus & &
\end{tabular}

Note. Total volume represents the number of voxels within an anatomically defined search region. Active volume represents the number of voxels within the region that exceed the significance threshold, $\propto=.05$ (one-tailed). Mean $Z$ score indicates the average of all suprathreshold voxels within the anatomical region. Stereotaxic coordinates and Maximum $Z$ score correspond to the voxel demonstrating the greatest signal change within an anatomical region.

traction map, performance-correlated activations were notably more focal and significant, suggesting that between-subject differences in encoding efficacy may contribute substantially to intersubject variability in functional activation.

The performance-related hemispheric effects that we report appear consistent with levels of processing effects reported in previous neuroimaging studies of verbal longterm memory (Kapur et al., 1994) and may correspond to differences in individual subjects' encoding strategies. The levels of processing framework (Craik \& Lockhart, 1972) suggests that items encoded deeply, according to their semantic attributes, are remembered more successfully than items encoded shallowly, according to their perceptual features. While this effect has been demonstrated robustly in behavioral studies (Craik \& Tulving, 1975), its neural correlates remain incompletely understood. Compared with shallow encoding, deep encoding of verbal stimuli has been found to preferentially engage left inferior frontal structures implicated in the retrieval of lexical semantic information and medial temporal structures implicated in the acquisition of word meanings (Kapur et al., 1996; Scoville, 1957; Wagner et al., 1998). It is possible that shallow encoding weakly activates the same structures engaged during deep encoding; alternatively, it is possible that shallow encoding also engages distinct neural structures. Our data support the latter possibility. In the present study, subjects were instructed to memorize stimulus sentences for a recognition memory test to follow, but the strategies they employed to do so were unconstrained, in contrast to previous studies (Fernandez et al., 1998, 1999). Participants were informally interviewed regarding their mnemonic strategies following scanning and behavioral testing. The subject with the highest discriminability score on the postscan recogni- 
tion test $(z=1.59)$ reported having tried to remember the (semantically unrelated) stimulus sentences by linking them together into a narrative. A subject whose discriminability score was among the lowest $(z=-0.80)$ reported having tried to "take a mental snapshot" of each stimulus, and two subjects who obtained discriminability scores slightly above the group mean $(z=0.16)$ reported having subvocally rehearsed each sentence until the next stimulus appeared. These three strategies correspond with semantic, orthographic, and phonological levels of processing which have been shown to promote high, low, and intermediate levels of subsequent recognition memory performance, respectively (Craik \& Tulving, 1975). Our observed left-hemisphere activation during successful encoding is consistent with deep semantic processing and with elaborative phonological rehearsal, both of which have been localized to left perisylvian structures (Paulesu, Frith, \& Frackowiak, 1993). Our finding of righthemisphere activation during relatively unsuccessful encoding is consistent with the results of a recent fMRI study that compared neural activity during orthographic, phonological, and lexicosemantic processing of visually presented words and found the greatest proportion of right-sided activation in occipital and temporal areas during orthographic processing (Fujimaki et al., 1999). No attempt was made to statistically correlate subjects' self-reported mnemonic strategies with their behavioral performance or functional activation, and we are cautious not to overinterpret these findings with respect to levels of processing effects. Nevertheless, these data provide impetus to explore the possibility that qualitatively different types of encoding may engage distinct neural structures.

Two recent studies have used event-related fMRI to investigate subsequent memory effects during deep and shallow verbal encoding tasks (Baker, Sanders, Macotta, \& Buckner, 2001; Otten, Henson, \& Rugg, 2001). These studies indicate that, in general, shallow encoding activates a subset of the frontal and medial temporal structures engaged during deep encoding and that subsequent memory effects during shallow encoding are found in a subset of structures that show subsequent memory effects during deep encoding. Although these results contrast with those of the present study, direct comparison is difficult due to numerous methodological differences: experimental design (blocked vs single-trial, and across-subject vs within-subject), stimuli (sentences vs words), and orienting tasks (intentional vs incidental encoding). Whereas Otten et al. (2001) analyzed only stimulus items that were correctly classified by the subject during encoding, our analyses included all encoding trials. Abstracting away from these differences in experimental design and analysis, however, the studies by Baker et al. (2001) and Otten et al. found that efficient and inefficient verbal encoding depend on overlapping neural structures, whereas our results indicate that efficient and inefficient verbal encoding engage distinct structures. Otten and colleagues note that their failure to find evidence of distinct structures associated with subsequent memory for shallowly encoded items constitutes a null result and should therefore be interpreted with caution. Further investigations may reveal that both distinct and overlapping neural structures subserve encoding that leads to relatively good and poor subsequent remembering.

\section{CONCLUSIONS}

Left- and right-hemisphere perisylvian structures were differentially engaged during relatively successful and unsuccessful verbal memory encoding. Subjects' self-reported encoding strategies suggest that performance-related differences in activation may correspond to different levels of processing. Future neuroimaging experiments that systematically vary encoding task requirements across subjects may 
further elucidate the relationship between the neural correlates of mnemonic strategies adopted at various levels of encoding and subsequent memory performance.

\section{REFERENCES}

Alsop, D. C. (1995). Correction of ghost artifacts and distortion in echoplanar MR with an iterative reconstruction technique. Radiology, 197P, 338.

Baker, J. T., Sanders, A. L., Macotta, L., \& Buckner, R. L. (2001). Neural correlates of verbal memory encoding during semantic and structural processing tasks. NeuroReport, 10(10), 1-7.

Casasanto, D. J., Killgore, W. D. S., Glosser, G., Maldjian, J. A., \& Detre, J. (2000). Hemispheric specialization during episodic memory encoding in the human hippocampus and MTL. Proceedings of the Cognitive Science Society, 22, 77-82.

Cohen, J. D., MacWhinney, B., Flatt, M., \& Provost, J. (1993). PsyScope: An interactive graphic system for designing and controlling experiments in the psychology laboratory using Macintosh computers; 22nd Annual Meeting of the Society for Computers in Psychology (1992, St. Louis, Missouri). Behavior Research Methods, Instruments, \& Computers, 25(2), 257-271.

Craik, F. I. M., \& Lockhart, R. S. (1972). Levels of processing: A framework for memory research. Journal of Verbal Learning and Verbal Behavior, 11, 671-684.

Craik, F. I. M., \& Tulving, E. (1975). Depth of processing and the retention of words in episodic memory. Journal of Experimental Psychology: General, 104, 268-294.

Fernandez, G., Brewer, J. B., Zhao, Z., Glover, G. H., \& Gabrielli, J. D. E. (1999). Level of sustained entorhinal activity correlates with subsequent cured-recall performance: A functional magnetic imaging study with high acquisition rate. Hippocampus, 9, 35-44.

Fernandez, G., Weyerts, H., Schrader-Bolsche, M., Tendolkar, I., Smid, H. G., Tempelmann, C., Hinrichs, H., Scheich, H., Elger, C. E., Mangun, G. R., \& Heinze, H. J. (1998). Successful verbal encoding into episodic memory engages the posterior hippocampus: a parametrically analyzed functional magnetic resonance imaging study. Journal of Neuroscience, 18(5), 1841-1847.

Fujimaki, N., Miyauchi, S., Putz, B., Sasaki, Y., Takino, R., Sakai, K., \& Tamada, T. (1999). Functional magnetic resonance imaging of neural activity related to orthographic, phonological, and lexicosemantic judgments of visually presented characters and words. Human Brain Mapping, 8(1), 4459.

Hinkle, D. E., Wiesrsma, W., \& Jurs, S. G. (1988). Applied statistics for the behavioral sciences. Boston: Houghton Mifflin.

Holmes, A., \& Friston, K. (1998). Generalizability, random effects \& population inference. NeuroImage, 7, S754.

Kapur, S., Craik, F. I., Tulving, E., Wilson, A. A., Houle, S., \& Brown, G. M. (1994). Neuroanatomical correlates of encoding in episodic memory: levels of processing effect [see comments]. Proceedings of the National Academy of Sciences of the USA, 91(6), 2008-2011.

Kapur, S., Tulving, E., Cabeza, R., McIntosh, A. R., Houle, S., \& Craik, F. I. (1996). The neural correlates of intentional learning of verbal materials: a PET study in humans. Brain Res Cognition Brain Research, 4(4), 243-249.

Kikinis, R., Gleason, P. L., Moriarty, T. M., Moore, M. R., Alexander, E., 3rd, Stieg, P. E., Matsumae, M., Lorensen, W. E., Cline, H. E., Black, P. M., \& Jolesz, F. A. (1996). Computer-assisted interactive three-dimensional planning for neurosurgical procedures. Neurosurgery, 38(4), 640-649; discussion 649-651.

Kirchhoff, B. A., Wagner, A. D., Maril, A., \& Stern, C. E. (2000). Prefrontal-temporal circuitry for episodic encoding and subsequent memory. Journal of Neuroscience, 20(16), 6173-6180.

Maldjian, J., Schulder, M., Liu, W., Mun, I., Hirschorn, D., \& Murthy, R. (1997). Intraoperative functional MRI using a real-time neurosurgical navigation system. Journal of Computed-Assisted Tomography, 21, 910-912.

Otten, L. J., Henson, R. N., \& Rugg, M. D. (2001). Depth of processing effects on neural correlates of memory encoding: Relationship between findings from across- and within-task comparisons. Brain, 124(2), 399-412.

Paulesu, E., Frith, C. D., \& Frackowiak, R. S. (1993). The neural correlates of the verbal component of working memory [see comments]. Nature, 362(6418), 342-345.

Scoville, W. B. (1957). Loss of recent memory after bilateral hippocampal lesions. J. Neurology and Neurosurgery in Psychiatry, 20, 11-21. 
Talairach, J., \& Tourneaux, P. (1988). Co-planar stereotaxic atlas of the human brain. New York: Thieme.

Tulving, E., Habib, R., Nyberg, L., Lepage, M., \& McIntosh, A. (1999). Positron emission tomography correlations in and beyond the medial temporal lobes. Hippocampus, 9, 71-82.

Tulving, E., Kapur, S., Markowitsch, H. J., Craik, F. I., Habib, R., \& Houle, S. (1994). Neuroanatomical correlates of retrieval in episodic memory: Auditory sentence recognition [see comments]. Proceedings of the National Academy of Sciences of the USA, 91(6), 2012-2015.

Wagner, A. D., Koutstaal, W., \& Schacter, D. L. (1999). When encoding yields remembering: insights from event-related neuroimaging. Philosophy Transcripts of the Royal Society of London B, Biological Sciences, 354(1387), 1307-1324.

Wagner, A. D., Schacter, D. L., Rotte, M., Koutstaal, W., Maril, A., Dale, A. M., Rosen, B. R., \& Buckner, R. L. (1998). Building memories: Remembering and forgetting of verbal experiences as predicted by brain activity. Science, 281(5380), 1188-1191. 